\title{
Accelerated Shoulder Rehabilitation after Reverse Total Shoulder Arthroplasty for Atraumatic Osteoarthritis: Study Protocol for a Single Center Randomized Controlled Trial
}

\author{
Avais Raja ( $\square$ avais.raja@tria.com ) \\ TRIA Research Institute https://orcid.org/0000-0003-2427-7808 \\ Travis Cambronne \\ TRIA Research Institute \\ Michael R Walsh \\ TRIA Research Institute
}

Study protocol

Keywords: Rehabilitation, Reverse Shoulder Arthroplasty, Accelerated

Posted Date: May 2nd, 2019

DOl: https://doi.org/10.21203/rs.2.9439/v1

License: (c) (i) This work is licensed under a Creative Commons Attribution 4.0 International License.

Read Full License 


\section{Abstract}

Background Reverse total shoulder arthroplasty has been successful in patients suffering from advanced degenerative osteoarthritis with rotator cuff insufficiency. The currently practiced physical rehabilitation after a shoulder rehabilitation does not allow shoulder motion until 6 weeks post-operatively. These patients are required to be in a sling to assist the patient in immobilizing the operated shoulder. An accelerated rehabilitation with immediate active shoulder motion has not been studied in the setting of a revere total shoulder arthroplasty. Methods This is a single center and single surgeon operated, randomized controlled trial. The primary objective is to compare patient reported shoulder function using the American Shoulder and Elbow Society (ASES) score and shoulder range of motion in patients undergoing the traditional physical rehabilitation and accelerated shoulder rehabilitation post reverse total shoulder arthroplasty. Secondary outcomes will include the Simple Shoulder Test (SST) score, Patient Reported Outcome Information System (PROMIS) Global Health-10, radiological parameters and subscapularis tear on ultrasound. Discussion The study will assess the effectiveness of an accelerated shoulder rehabilitation with immediate active shoulder motion after a reverse total shoulder arthroplasty in patients with atraumatic osteoarthritis.

\section{Background}

Reverse Total Shoulder Arthroplasty (RTSA) initially termed by Grammont et al in 1993, was originally designed to treat rotator cuff arthropathy. ${ }^{1}$ With the success of this procedure, orthopedic surgeons have extended its indication to rotator cuff arthropathy. RTSA is now utilized to treat some complex proximal humeral fractures, massive irreparable rotator cuff tears, and shoulder arthroplasty revision surgery. ${ }^{2,3}$ Patients that have undergone RTSA have exhibited great improvement in quality of life and activities due to enhancement of function and pain relief. ${ }^{4}$ Long term results have been reported and continue to be evaluated. ${ }^{5}$ From an economic standpoint, studies have shown RTSA to be more cost-effective than Total Shoulder Arthroplasty (TSA) and Hemi-arthroplasty (HA) with respect to cost of implant and low rate of complication from the surgery. ${ }^{6-8}$

Anecdotally, we have noticed patients with a complaint of limited range of motion at the end of one year possibly due to the delay in the onset of shoulder motion. Attention to the timing and method of postoperative rehabilitation following reverse arthroplasty may be important, given that many of these patients are elderly, need early independence, may be more unsteady on their feet if immobilized, and are in need of variable amounts of post-operative assistance. The post-operative rehabilitation for RTSA is variable, and may depend on patient needs, intra-operative pathology, implant stability, and surgeon preference. Some surgeons practice a period of immobilization for 2-6 weeks while others begin immediate active range of motion to wean the patient from the sling. ${ }^{9} \mathrm{~K}$ waees et al, conducted a Delphi consensus study on managing patients who are to undergo a RTSA, where ten orthopedic surgeons responded differently when it came to the rehabilitation protocols. ${ }^{10,11}$ They found great disparity in postoperative rehabilitation protocols. 
A reverse total shoulder arthroplasty protocol has been published over a decade ago. ${ }^{12}$ This protocol outlines a joint protection phase for the shoulder for a period of 6 weeks without any shoulder active range of motion (AROM) and sling use for upto 4-6 weeks. ${ }^{12}$ At the moment, we are not aware of any literature studying an immediate active shoulder rehabilitation (IASR) after a RTSA.

We propose an immediate accelerated active shoulder physical therapy protocol with less need for outpatient therapy visits that potentially offers more rapid return to independence, increased satisfaction, and increased value to the patient. We hypothesize that this post-operative approach to rehabilitation is safe with superior shoulder functional outcomes and reduction in overall cost.

\section{Methods}

\section{Overview}

This is single center randomized controlled trial based on an alpha-adaptive design and a 1:1 allocation ratio. The study compares shoulder functional outcomes, complications, subscapularis muscle integrity and number of physical therapy (PT) visits between two different postoperative shoulder physical therapy protocols. The study will be conducted at an outpatient orthopaedic center affiliated with an inpatient surgery center. The diagnosis of rotator cuff arthropathy will be made by the orthopaedic surgeon and the decision to proceed with a reverse total shoulder arthroplasty will be determined by a consensus between the patient and surgeon. After the surgery, the patient will either follow the standard physical therapy protocol or the immediate accelerated protocol. Follow up visits will be performed at 2 weeks, 6 weeks, 3 months, 6 months, and 1 year after the surgery. Based on previous volume of reverse total shoulder arthroplasty in the preceding years, we expect the study to run for 3 years, with 2 years of recruitment and 1 year of follow-up.

\section{Study Population}

Inclusion Criteria

- 55 years of age.

- Candidate for a primary reverse total shoulder arthroplasty. (CPT code 23472)

- Capable of completing self-administered questionnaires.

- Be willing and able to return for all study-related follow-up procedures.

- Able and willing to give informed consent.

- Proficient in the English language. 
In-Clinic

- Patients planning on undergoing a Primary Reverse Shoulder Total Arthroplasty due to proximal humeral fracture.

- Previous shoulder surgery including previous total shoulder arthroplasty or reverse total shoulder arthroplasty or hemiarthroplasty, or instability repairs

- Active bacterial infection of the shoulder.

- Any concomitant shoulder procedure.

- Additional ipsilateral or contralateral upper limb pathology that requires active treatment (i.e. surgery or brace).

- Peripheral vascular disease or other vascular disorders that would impair healing.

- Peripheral neuropathy or other neurological disorders that may impair the patient to ambulate.

- Patient is on workers compensation.

- Any condition requiring chemotherapy.

- Active tobacco user or former tobacco user who is not free of using tobacco for 8 weeks.

- Uncontrolled Diabetes Mellitus with an $\mathrm{HbA} 1 \mathrm{C}>7.5 \%$.

- Current drug or alcohol abuse.

- Major medical illness (life expectancy less than 2 years or unacceptably high operative risk)

- Suspicion of cervical radiculopathy or myelopathy.

- Deltoid insufficiency on physical examination

Intra-operative:

- latrogenic glenoid fracture

- Post-operative:

- Neurological injury of the upper extremity.

- Complications from Primary Reverse Shoulder Total Arthroplasty (i.e. post-operative infection, bleeding, hardware failure).

\section{Randomization}

Patients will be randomly be assigned to the individual post-operative physical therapy group using a computerized random number generator. A block randomization scheme and an allocation ratio of 1:1 will be employed. The allocation sequence will be concealed in sealed envelopes and revealed after the surgery is performed. The randomization will be conducted by the manager of clinical research. Participants will be enrolled by a post-doctoral research fellow. The investigator will assign participants to the intervention and will be documented in the operative note and post-operative orders to make the 
physical therapists and nurses aware. The outcome assessor for range of motion and data analyst will be blinded after the intervention is assigned to the participant.

\section{Interventions}

Subjects in the immediate active shoulder rehabilitation group will be provided with a physical therapy home sheet. During their inpatient stay at the hospital post-operatively for 1-3 days, the physical therapist will teach and practice several stretching exercises with the subjects. The exercises include: elbow flexion/extension, forearm rotation, pendulum, wand external rotation, standing passive external rotation, supine assisted flexion range of motion (ROM), table slide flexion, scapular retraction, adduction, internal rotation, flexion, abduction with external rotation, external rotation on wall, and posterior capsular stretch. Upon discharge of the patient from the hospital, the physical therapist will specify the sets, repetitions, number of times per day and seconds to hold for each exercise. Additional outpatient physical therapy visits may be ordered if the patient has difficulty in performing the exercises or requests for it.

The patients in the standard group will follow what is the current standard of healthcare, which is a total of 18 outpatient physical therapy visits in a time span of 12 weeks. There will be 2 visits per week for the first 6 weeks followed by 1 visit per week for the next 6 weeks. The patient will be in a shoulder sling for 46 weeks and restricted from active range of motion (AROM) of the shoulder. During the first 6 weeks the patients will learn and practice the following exercises: passive range of motion of the shoulder in the scapular plane, active range of motion of the elbow, wrist, and hand, pendulum movements, scapular exercises (scapular retraction, depression, circles), and deltoid isometrics. In the last 6 weeks, the patient will begin active assisted range of motion and active range of motion of the shoulder, progress with the scapular and glenohumeral stabilization exercises. At week 10, exercises will progress from isometric to isotonic.

\section{Outcomes}

The primary outcome of this study is shoulder function measured by questionnaires and range of motion. Patient reported outcome measures used for this study is the American Shoulder and Elbow Score (ASES) and Simple Shoulder Test (SST) score. Range of motion will be measured with the help of a goniometer for forward elevation, external rotation and internal rotation. The measurements will be performed by a blinded orthopaedic surgeon fellow with at least 5 years of postgraduate clinical training experience. These will be collected at every visit: baseline, post-operative 2 weeks, 6 weeks, 3 months, 6 months, and 1 year. (Figure 1)

The secondary outcome is serial shoulder radiographs in the anteroposterior and lateral views to detect endoprosthesis loosening, scapular notching, and dislocation. Radiographs will be performed at every visit as well. 
The tertiary outcome is a one-time shoulder ultrasound performed by a blinded musculoskeletal radiologist at the end of 1 year follow up to detect a tear of the subscapularis muscle.

The fourth outcome is number of outpatient physical therapy visits required for the shoulder.

Sample Size

The sample size calculation was based on the postoperative ASES score in a comparative study published by other authors. ${ }^{13}$ To detect a clinically relevant difference of 15.8 score between groups, with a mean improvement SD of 20.6, alpha of $5 \%$ and power of $80 \%, 31$ patients were needed in each group. Assuming a drop-out rate of $20 \%$, at least 37 patients were required per group. This leaves us with a total sample size of 74 .

If we consider the minimal clinical important difference (MCID) of 3.0 for the Simple Shoulder Test score, we would need 19 patients per group, respectively to possibly reach statistical significance in these outcome scores. A total of 34 patients will be necessary to detect a $10^{\circ}$ difference in range of motion of the shoulder in all planes based on an SD of $10^{\circ}$.

\section{Recruitment}

The participants will be recruited from an outpatient orthopaedic surgery clinic. If a participant tentatively meets the criteria for the study, the investigator will inform the research fellow/coordinator to complete the recruitment process. Patients who are willing to participate in the randomized trial will be asked to sign a Consent Form. Basic demographic data will be collected for both groups to assess independent variables (age, gender, BMI, hand dominance, race, ethnicity, type of osteoarthritis baseline shoulder function (American Shoulder and Elbow Society (ASES) score, SST score and PROMIS Global Health-10, and baseline shoulder range of motion..

\section{Data Collection Methods}

Data on outcomes will be collected on a hardcopy format and transferred to a data collection software, REDCap. Case report forms will be provided to the research coordinator and radiologist to document findings. Surveys on patient reported shoulder function and general health will be completed at each visit on paper as well.

Statistical Analysis 
Differences in baseline characteristics (i.e., intrinsic and injury-related variables) between both intervention groups will be assessed using the Student' s t test (parametric continuous data), the MannWhitney U test (nonparametric continuous data) or the chi-square test (categorical data). Unadjusted analysis by intention-to-treat will be performed to test the difference in primary and secondary outcomes between the intervention groups. Student' s t test (parametric continuous data), the Mann-Whitney U test (nonparametric continuous data) or the chi-square analysis (categorical data) will be used. A p value < 0.05 (two-sided tests) will be taken as threshold of statistical significance.

\section{Monitoring and Quality Assurance}

The institutions Data and Safety Monitoring Board (DSMB) will review the progress of the study to assure the rights and safety of participants, confirm the study conduct follows the guidelines of good clinical practice, assure maintenance of required documents, verify adherence to the protocol, monitor the quality of data collected, and assure accurate reporting and documentation of all adverse events. Research auditing will occur independently from the investigators.

Adverse events will be collected and recorded on the appropriate case report until the patient participation is complete at the 1 year post-operative time point. Adverse events will be noted subjectively from the patient or objectively during the follow-up visits.

All research documentation will be labelled with a unique person number as an identifier and stripped of any potentially identifiable information. Data will be stored in password-protected computers and locked filing cabinets as required within the administering institution.

\section{Ethics and Dissemination}

IRB approval was obtained from the HealthPartners Institute on October 18, 2018. Protocol modifications, addition of staff personal and changes to the consent form will be presented to the IRB as an amendment. Consent will be obtained by the research fellow or coordinator.

The study received full internal funding through the HealthPartners Institute via competitive process throughout all medical and surgical departments of the institute. The final trial dataset will be under the access of the research fellow/coordinator and statistician. There is no plans of sharing the data set and statistical analysis reports to the public.

Our plan is to present the results of our study via publication and abstract presentation. We initially plan to submit our study protocol to the BMC Trials Journal as a manuscript after IRB approval and before we begin recruitment to showcase our study to prevent duplicate work by other centers and creating an opportunity for other shoulder surgeons to provide constructive criticism to improve the study. On 
completion of final data analysis, an abstract will be submitted to appropriate shoulder conference i.e. American Shoulder and Elbow Society. Following this, we will write up our results in the form of a manuscript for journal submission.

\section{Discussion}

The deltoid becomes the primary muscle required for shoulder movement after a reverse total shoulder arthroplasty. An accelerated rehabilitation was studied for anatomical shoulder arthroplasty with maximal improvements in range of motion and patient reported shoulder function. ${ }^{14}$ However, there are no randomized controlled trials comparing an accelerated rehabilitation to the traditional rehabilitation after a reverse total shoulder arthroplasty.

This proposed trial will compare multiple outcomes to outline the safety and advantage of allowing early active shoulder motion from this procedure. The subscapularis muscle will be repaired deeming it necessary to evaluate the integrity of the repair. We plan to assess the subscapularis using an ultrasound and comparing the tear rates between both rehabilitation groups.

The major benefit of this study is the ability to move the shoulder early which is important in the elderly population, most of them requiring assistance already. Early motion may also help decrease the problem of stiffness observed after the disuse of the shoulder from the surgery.

\section{Trial Status}

Patient recruitment is currently open. Recruitment began in January 2019 and is expected to end by December 2020. The protocol that is being followed is version 6.0, dated on 01/10/2019.

\section{Abbreviations}

- American Shoulder and Elbow Society (ASES)

- Simple Shoulder Test (SST)

- Patient Reported Outcome Information System (PROMIS)

- Reverse Total Shoulder Arthroplasty (RTSA)

- Total Shoulder Arthroplasty (TSA)

- Hemi-arthroplasty (HA)

- Immediate active shoulder rehabilitation (IASR)

- physical therapy (PT)

- range of motion (ROM),

- Active range of motion (AROM)

- minimal clinical important difference (MCID) 
- Data and Safety Monitoring Board (DSMB)

\section{Declarations}

\section{Ethics Approval and Consent to Participate}

The study was approved by HealthPartners Institutional Review Board. A written informed consent will be obtained from all participants.

\section{Consent for Publication}

Not applicable.

Availability of Data and Materials

Data and materials will be available after completion of the study.

\section{Funding}

The study is currently funded by HealthPartners Institute Internal Grant. The funding will cover study indirect costs i.e. coordinator time, radiologist time, administrative time, statistician time and direct costs i.e. 2 additional shoulder radiographs, 1 shoulder ultrasound and patient stipend. It will not cover for data interpretation and writing the manuscript.

\section{Competing Interests}

There are no competing interests for this study.

\section{Authors Contributions}

All the authors were involved in study idea inception, protocol write-up, IRB submission, and patient recruitment.

Study Inception/Design: AR, TC, and MRW

Protocol Write-up: AR, TC, and MRW 
IRB Submission: AR

Patient Recruitment: AR, TC, and MRW

Acknowledgments

There are no acknowledgements.

\section{References}

1. Grammont PM, Baulot E. Delta shoulder prosthesis for rotator cuff rupture. Orthopedics. 1993 Jan 1;16(1):65-8.

2. Young AA, Smith MM, Bacle G, et al. Early results of reverse shoulder arthroplasty in patients with

3. Rheumatoid arthritis. J Bone Joint Surg Am 2011; 93:1915-23.

4. Wall B, Nové-Josserand L, O'Connor DP, Edwards TB, Walch G. Reverse total shoulder arthroplasty: a review of results according to etiology. J Bone Joint Surg Am. 2007 Jul 1;89(7):1476-85.

5. Bacle G, Nové-Josserand L, Garaud P, Walch G. Long-term outcomes of reverse total shoulder arthroplasty: a follow-up of a previous study. JBJS. 2017 Mar 15;99(6):454-61.

6. Schairer WW, Nwachukwu BU, Lyman S, Craig EV, Gulotta LV. Reverse shoulder arthroplasty versus hemiarthroplasty for treatment of proximal humerus fractures. Journal of shoulder and elbow surgery. 2015 Oct 1;24(10):1560-6.

7. Osterhoff G, O'Hara NN, D'Cruz J, Sprague SA, Bansback N, Evaniew N, Slobogean GP. A costeffectiveness analysis of reverse total shoulder arthroplasty versus hemiarthroplasty for the management of complex proximal humeral fractures in the elderly. Value in Health. $2017 \mathrm{Mar}$ 1;20(3):404-11.

8. Boileau P, Watkinson D, Hatzidakis AM, Hovorka I. Neer Award 2005: The Grammont reverse shoulder prosthesis: results in cuff tear arthritis, fracture sequelae, and revision arthroplasty. Journal of shoulder and elbow surgery. 2006 Oct 31;15(5):527-40.

9. Coe MP, Greiwe RM, Joshi R, Snyder BM, Simpson L, Tosteson AN, Ahmad CS, Levine WN, Bell JE. The cost-effectiveness of reverse total shoulder arthroplasty compared with hemiarthroplasty for rotator cuff tear arthropathy. Journal of shoulder and elbow surgery. 2012 Oct 31;21(10):1278-88.

10. Jarrett CD, Brown BT, Schmidt CC. Reverse shoulder arthroplasty. Orthopedic Clinics of North America. 2013 Jul 31;44(3):389-408.

11. Kwaees TA, Charalambous CP. Reverse Shoulder Arthroplasty-Minimum Age for Surgery, Postoperative Rehabilitation and Long Term Restrictions. A Delphi Consensus Study. Ortopedia, traumatologia, rehabilitacja. 2013 Dec;16(4):435-9. 
12. Boudreau S, Boudreau ED, Higgins LD, Wilcox III RB. Rehabilitation following reverse total shoulder arthroplasty. journal of orthopaedic \& sports physical therapy. 2007 Dec;37(12):734-43.

13. Simovitch R, Flurin PH, Wright T, Zuckerman JD, Roche CP. Quantifying success after total shoulder arthroplasty: the minimal clinically important difference. Journal of shoulder and elbow surgery. 2018 Feb 1;27(2):298-305.

14. Denard PJ, Lädermann A. Immediate versus delayed passive range of motion following total shoulder arthroplasty. Journal of shoulder and elbow surgery. 2016 Dec 1;25(12):1918-24.

\section{Figures}

\begin{tabular}{|c|c|c|c|c|c|c|c|c|c|}
\hline \multirow[b]{3}{*}{ TIMEPOINT $^{\star *}$} & \multicolumn{9}{|c|}{ STUDY PERIOD } \\
\hline & \multirow{2}{*}{$\begin{array}{c}\text { Enrolment } \\
-t_{1} \\
\end{array}$} & \multirow{2}{*}{$\begin{array}{c}\text { Allocation } \\
0\end{array}$} & \multirow[b]{2}{*}{$\begin{array}{l}\text { Post- } \\
\text { RTSA }\end{array}$} & \multicolumn{5}{|c|}{ Post-allocation } & \multirow{2}{*}{$\begin{array}{c}\text { Close-out } \\
1 \mathrm{yr} \\
\end{array}$} \\
\hline & & & & $\begin{array}{c}2 \\
w k s \\
\end{array}$ & $\begin{array}{c}6 \\
w k s \\
\end{array}$ & $\begin{array}{c}3 \\
\text { mon } \\
\end{array}$ & $\begin{array}{c}6 \\
\text { mon } \\
\end{array}$ & $1 \mathrm{yr}$ & \\
\hline \multicolumn{10}{|l|}{ ENROLMENT: } \\
\hline \multirow{2}{*}{$\begin{array}{l}\text { Eligibility screen } \\
\text { Informed consent }\end{array}$} & $\mathrm{x}$ & & & & & & & & \\
\hline & $\mathrm{x}$ & & & & & & & & \\
\hline Allocation & & $x$ & & & & & & & \\
\hline \multicolumn{10}{|l|}{ INTERVENTIONS: } \\
\hline $\begin{array}{r}\text { Accelerated } \\
\text { Rehabilitation }\end{array}$ & & & $\mathrm{x}$ & & & & & & \\
\hline $\begin{array}{r}\text { Traditional } \\
\text { Rehabilitation }\end{array}$ & & & $\mathrm{x}$ & $\leftarrow$ & & & & & \\
\hline \multicolumn{10}{|l|}{ ASSESSMENTS: } \\
\hline \multirow{2}{*}{$\begin{array}{r}\text { Demographic } \\
\text { Details } \\
\text { Patient Reported } \\
\text { Outcome: ASES, } \\
\text { SST, PROMIS-10 }\end{array}$} & $\mathrm{x}$ & & & & & & & & \\
\hline & $\mathrm{x}$ & & & $\mathrm{x}$ & $\mathrm{x}$ & $\mathrm{X}$ & $\mathrm{X}$ & $\mathrm{X}$ & $\mathrm{x}$ \\
\hline Shoulder ROM & $\mathrm{x}$ & & & & & $x$ & $x$ & $x$ & $x$ \\
\hline $\begin{array}{r}\text { Shoulder } \\
\text { Radiographs }\end{array}$ & & & & $\mathrm{x}$ & $x$ & $x$ & $x$ & $x$ & $x$ \\
\hline $\begin{array}{r}\text { Shoulder } \\
\text { Ultrasound }\end{array}$ & & & & & & & & $x$ & $x$ \\
\hline
\end{tabular}

Figure 1 
Standard Protocol Items: Recommendations for Interventional Trials (SPIRIT) diagram.

\section{Supplementary Files}

This is a list of supplementary files associated with this preprint. Click to download.

- SPIRITFillablechecklistRTSARehabv1.022JAN2019.doc 